\title{
Dexamethasone-mediated oncogenicity in vitro and in an animal model of glioblastoma
}

\author{
Markus M. Luedi, MD, MBA,, ${ }^{1,2}$ Sanjay K. Singh, PhD, ${ }^{2}$ Jennifer C. Mosley, MSc, ${ }^{2}$ \\ Islam S. A. Hassan, MD, ${ }^{2}$ Masumeh Hatami, MD, ${ }^{2}$ Joy Gumin, BSc, ${ }^{3,4}$ Lukas Andereggen, MD, ${ }^{5-7}$ \\ Erik P. Sulman, MD, PhD, ${ }^{8}$ Frederick F. Lang, MD, ${ }^{3,4}$ Frank Stueber, MD, ${ }^{1}$ \\ Gregory N. Fuller, MD, PhD, ${ }^{9}$ Rivka R. Colen, MD, ${ }^{2}$ and Pascal O. Zinn, MD, PhD $2,3,10,11$
}

Departments of ${ }^{1}$ Anesthesiology and ${ }^{7}$ Neurosurgery, Bern University Hospital Inselspital, University of Bern, Switzerland; Departments of ${ }^{2}$ Cancer Systems Imaging, ${ }^{3}$ Neurosurgery, ${ }^{8}$ Radiation Oncology, ${ }^{9}$ Pathology, and ${ }^{11}$ Cancer Biology, and ${ }^{4}$ Brain Tumor Center, The University of Texas MD Anderson Cancer Center, Houston; ${ }^{10}$ Department of Neurosurgery, Baylor College of Medicine, Houston, Texas; ' Department of Neurosurgery and the F.M. Kirby Neurobiology Center, Boston; and ${ }^{6}$ Children's Hospital and Harvard Medical School, Boston, Massachusetts

\begin{abstract}
OBJECTIVE Dexamethasone, a known regulator of mesenchymal programming in glioblastoma (GBM), is routinely used to manage edema in GBM patients. Dexamethasone also activates the expression of genes, such as CEBPB, in GBM stem cells (GSCs). However, the drug's impact on invasion, proliferation, and angiogenesis in GBM remains unclear. To determine whether dexamethasone induces invasion, proliferation, and angiogenesis in GBM, the authors investigated the drug's impact in vitro, in vivo, and in clinical information derived from The Cancer Genome Atlas (TCGA) cohort.
\end{abstract}

METHODS Expression profiles of patients from the TCGA cohort with mesenchymal GBM $(n=155)$ were compared with patients with proneural GBM by comparative marker selection. To obtain robust data, GSCs with IDH1 wild-type (GSC3) and with IDH1 mutant (GSC6) status were exposed to dexamethasone in vitro and in vivo and analyzed for invasion (Boyden chamber, human-specific nucleolin), proliferation (Ki-67), and angiogenesis (CD31). Ex vivo tumor cells from dexamethasone-treated and control mice were isolated by fluorescence activated cell sorting and profiled using Affymetrix chips for mRNA (HTA 2.0) and microRNAs (miRNA 4.0). A pathway analysis was performed to identify a dexamethasone-regulated gene signature, and its relationship with overall survival (OS) was assessed using KaplanMeier analysis in the entire GBM TCGA cohort $(n=520)$.

RESULTS The mesenchymal subgroup, when compared with the proneural subgroup, had significant upregulation of a dexamethasone-regulated gene network, as well as canonical pathways of proliferation, invasion, and angiogenesis. Dexamethasone-treated GSC3 demonstrated a significant increase in invasion, both in vitro and in vivo, whereas GSC6 demonstrated a modest increase. Furthermore, dexamethasone treatment of both GSC3 and GSC6 lines resulted in significantly elevated cell proliferation and angiogenesis in vivo. Patients with mesenchymal GBM had significant upregulation of dexamethasone-regulated pathways when compared with patients with proneural GBM. A prognostic $(p=0.0007)$ 33-gene signature was derived from the ex vivo expression profile analyses and used to dichotomize the entire TCGA cohort by high (median OS 12.65 months) or low (median OS 14.91 months) dexamethasone signature.

CONCLUSIONS The authors present evidence that furthers the understanding of the complex effects of dexamethasone on biological characteristics of GBM. The results suggest that the drug increases invasion, proliferation, and angiogenesis in human GSC-derived orthotopic tumors, potentially worsening GBM patients' prognoses. The authors believe that careful investigation is needed to determine how to minimize these deleterious dexamethasone-associated side effects in GBM.

https://thejns.org/doi/abs/10.3171/2017.7.JNS17668

KEY WORDS glioblastoma; glioblastoma stem cells; dexamethasone; proliferation; angiogenesis; invasion; oncology 
$\mathrm{S}$ EVERAL important hallmarks of cancer, such as sustained proliferative signaling, replicative immortality, evasion of growth suppressors, resistance to cell death, activation of invasion, metastasis, and angiogenesis, have been described., ${ }^{5,6}$ Glioblastoma (GBM) is the most aggressive and most common primary brain tumor in adults. ${ }^{26}$ It is characterized by abundant and aberrant neovascularization, invasion of tumor cells into neighboring normal brain parenchyma, and a high proliferation index; specifically, angiogenesis is used to elevate the diagnosis from Grade III astrocytoma to Grade IV, i.e., GBM. T Tumor neovascularization causes cerebral edema, mass effect, and neurological deficit, which often affects quality of life $\mathrm{e}^{10}$ and often contributes to low survival durations of 15.3-21.7 months. ${ }^{8}$ Worldwide, patients with GBM-associated vasogenic edema are for the most part treated with dexamethasone throughout the treatment course.

Different molecular subclasses of GBM have been described. ${ }^{24}$ The mesenchymal subtype, which is partially controlled by the transcription factor CEBPB, ${ }^{2}$ is the most aggressive GBM; the proneural subtype, which is partially characterized by an isocitrate dehydrogenase 1 gene (IDHI) mutation, is the least aggressive. ${ }^{19,24} \mathrm{IDHI}$ has been shown to be mutated in $12 \%$ of GBM patients ${ }^{16}$ most commonly in secondary, less vascularized tumors ${ }^{16,28}$ that have been reported to confer a better patient prognosis (mean 27.1 months vs 11.3 months). ${ }^{15}$ However, as part of standard practice, all patients, irrespective of molecular subtype, are similarly treated with dexamethasone, surgery if indicated, temozolomide, and radiation therapy.

Cancer stem cells play a key role in GBM and are termed glioblastoma stem cells (GSCs). ${ }^{21,22}$ GSCs are a reliable tool for recapitulating the disease's biological characteristics. ${ }^{2}$ Recently, our in vitro study showed that a dexamethasone-regulated gene signature activates $C E B P B$ expression in $\mathrm{GSCs}^{11}$ and thus demonstrated that dexamethasone treatment can have adverse implications on the molecular biological characteristics of GBM and on the treatment efficiency conferred by standard-of-care therapy ${ }^{27}$ However, the drug's impact on invasion, proliferation, and angiogenesis in GBM remains unclear. To determine whether dexamethasone induces invasion, proliferation, and angiogenesis in GBM, we investigated the drug's impact in vitro using GSCs, in vivo using GSCderived orthotopic tumors in mice, and in clinical information obtained from The Cancer Genome Atlas (TCGA) database.

\section{Methods \\ Institutional Approval}

The GSC lines were derived from patients after obtaining written informed consent under The University of Texas MD Anderson Cancer Center (Houston, Texas) institutional review board-approved protocol. All mouse experiments were performed in accordance with institutional and governmental laws and standards, and were approved by the MD Anderson institutional Animal Care and Use Committee in accordance with the American Association for Laboratory Animal Science guidelines.

\section{Identification of Drugs Predicted to Be Upstream Regulators in Mesenchymal Versus Proneural Genomic Analysis}

We downloaded gene expression level and overall survival data from 520 patients with GBM in the TCGA database. From this large cohort of patients, the gene expression levels of the mesenchymal $(\mathrm{n}=155)$ and proneural ( $n=99$ ) subtypes were obtained. A comparative marker selection module of GenePattern software from the Broad Institute was used to obtain fold changes, $p$ values, and false discovery rates (FDRs) to identify genes that are differentially expressed between the mesenchymal and proneural subtypes. Gene lists with fold change cutoffs of 5, 6 , and 8 were analyzed using Ingenuity Pathway Analysis (IPA; Qiagen) software to identify 1) drugs and chemical compounds with significantly altered downstream genes/ networks and 2) significantly altered cellular functions.

\section{Tissue Samples and Stem Cell Cultures}

Tissue from GBM patients was collected intraoperatively at MD Anderson and processed after histopathological confirmation, as performed previously. ${ }^{11}$ GBM patient-derived GSCs were cultured as described previously. ${ }^{9,11,22}$ In brief, tumors were isolated, followed by enzyme treatment to obtain a single cell suspension that was then cultured in GSC media (DMEM/F12 containing 1× B27, $20 \mathrm{ng} / \mathrm{ml}$ EGF, and $20 \mathrm{ng} / \mathrm{ml}$ basic fibroblast growth factor). Two independent GSC lines (GSC3 and GSC6) were cultured as neurospheres for further scrutiny. For this study, we selected an IDHI wild-type and a IHDI mutant GSC line.

\section{Western Blot Analysis of IDH1 Status}

Whole cell extracts from in vitro cultures were analyzed by Western blot analysis according to standard protocols. The membranes of IDHI mutant and wild-type cells were probed with mouse anti-IDH1 R132H (HistoBioTec) and rat anti-IDH1 (HistoBioTec) primary antibodies at $4^{\circ} \mathrm{C}$ overnight to determine IHD1 status. Rat anti-IDH1 wildtype cells also served as a loading control. Membranes were incubated with species-specific horseradish peroxidase-conjugated secondary antibodies for 1 hour at ambient temperature, exposed to Clarity Western enhanced chemiluminescence substrate (BioRad), and scanned using a c600 Imaging System (Azure Biosystems), or an immunofluorescent IRDye 800CM secondary antibody against rat (Li-Cor), scanned directly at $800 \mathrm{~nm}$ after secondary incubation.

\section{Confirmation of Orthotopic Tumor Formation}

Six 5-week-old male nude mice (stain nu/nu, obtained from the Department of Veterinary Medicine and Surgery, MD Anderson) were anesthetized with intraperitoneal ketamine;, 911 a nylon bolt was implanted in orthotopic position (right forebrain), as described previously. Seven days after bolt implantation, $1 \times 10^{5}$ human-derived GSC3s and GSC6s in $5 \mu$ l of DMEM were injected in the orthotopic position in 2 groups of 3 mice via the bolts over a 5-minute period. Mice were killed at the earliest onset of neurological symptoms using hypercarbia and decapitation, according to the animal protocol. 


\section{In Vitro Model}

For the in vitro studies, we used $50 \mu \mathrm{M}$ dexamethasone, a concentration that was within the wide range that was previously described for in vitro studies. ${ }^{1,11}$ Neurospheres were exposed to Accutase cell detachment solution (EMD Millipore) for 3 minutes to obtain single cells and then cultured in GSC medium containing $50 \mu \mathrm{M}$ dexamethasone (Sigma) for 6 days or GSC medium alone for the control, with media exchanged daily.

\section{In Vivo Model}

After the orthotopic tumor formation capability of both GSCs had been confirmed, 10 mice were injected with 1 $\times 10^{5}$ human GSC3, and another 10 were injected with GSC6, as described above. Starting on the day of xenotransplantation, mice were treated daily with $2 \mathrm{mg} / \mathrm{kg}(50$ $\mu \mathrm{g}$ in $100 \mu \mathrm{l}$ of $\mathrm{H}_{2} \mathrm{O}$ ) dexamethasone (Sigma) intraperitoneally (controls, $100 \mu \mathrm{l}$ of $\mathrm{H}_{2} \mathrm{O}$ only intraperitoneally daily). ${ }^{11,12}$ After the mice were killed, their brains were immediately collected in formalin and embedded in paraffin or added to collection solution; live cells were processed with the NeuroCult enzyme dissociation kit (STEMCELL Technologies) according to manufacturer's protocol.

\section{Ex Vivo Isolation of Human GSC-Derived Orthotopically Grown Tumor Cells}

Tumors from multiple mice in the GSC3 and GSC6 groups were pooled and live cells underwent fluorescenceactivated cell sorting (FACS) using rat anti-human-HLA class I ABC antibodies (Acris) and secondary allophycocyanin (APC) goat anti-rat (BD Pharmingen) antibody. Briefly, the positive gates for cell sorting were determined based on cells labeled with isotype-negative control antibody (anti-rat immunoglobulin G), where forward scatter absorption (FSC-A) on the $\mathrm{x}$-axis was used as a guide for single cells, and APC-A (allophycocyanin absorption) on the y-axis was used to detect APC-positive human cells. Isolated human GSC-derived cells were used for total RNA extraction (mRNA and microRNA [miRNA]).

\section{RNA Isolation and Whole Genome Expression Profiling}

RNA was isolated with PureZOL (Bio-Rad) following the manufacturer's protocol. To precipitate small RNA molecules such as miRNA, $10 \mu \mathrm{g}$ of linear acrylamide (Invitrogen) was added at the isopropanol precipitation step. After quality control had been performed, RNA was studied with human transcriptome 2.0 (Affymetrix) and miRNA 4.0 arrays (Affymetrix) according to the manufacturer's protocols. Probe cell intensity data (.cel files) were uploaded and normalized using Expression Console software (Affymetrix). The fold changes in the expression levels of genes were determined using the Transcriptome Analysis Console (Affymetrix). We used IPA to identify gene networks and their cellular function annotations, with $\mathrm{a} \pm 1.5$-fold change cutoff for both mRNA and miRNA, as described previously. ${ }^{3,11}$

\section{Gene Set Enrichment Analysis}

For the gene set enrichment analysis (GSEA), we used GSEA software with the current release of the molecular signature database (v5.2 MSigDB) from the Broad Institute. In brief, .gct files were created using gene signal intensity data for dexamethasone-treated and control samples (ex vivo). A GSEA analysis was performed with 1000 permutations of "Gene set" using a "h.all.v5.2.symbols. gmt" database. The enrichment plot and associated statistical values were obtained from the GSEA report for the data set.

\section{In Vitro and In Vivo Invasion Assays}

The in vitro cell invasion properties of GSC3 and GSC6 were characterized using Boyden Chamber assays (EMD Millipore) according to the manufacturer's guidelines. In brief, this assay has 2 chambers. Cells are seeded in the upper chamber, and chemoattractant is placed in the lower chamber. Hydrolyzing cells invade the extracellular matrix and are present on the other face of the membrane at the end of the experiment. GSCs were pretreated with $50 \mu \mathrm{M}$ dexamethasone for 5 days. On day $5,5 \times 10^{4}$ cells were seeded in inserts, while the wells contained either $10 \%$ fetal bovine serum alone (control) or $10 \%$ fetal bovine serum with $50 \mu \mathrm{M}$ dexamethasone (for dexamethasone pretreated cells). These cells were incubated for an additional 22 hours. Matrigel and noninvading GSCs within the inserts were removed; membranes were fixed in methanol and stained with $\mathrm{H} \& \mathrm{E}$ following a standard protocol. Stained membranes were then mounted on microscope slides and imaged using $20 \times$ or $40 \times$ microscope objectives. ImageJ software was used to count the GSC-derived cells that invaded through the Matrigel layer and are present on the other side of membrane. Statistical significance of the numbers of invading dexamethasonetreated and control GSCs from 2 biological duplicates and 8 technical replicates each were analyzed using Prism 6 software (GraphPad). The tumor cells were identified using human-specific nucleolin antibody (this antibody does not react to mouse antigen; thus, it selectively labels cells of human origin). The tumor margin/edge was defined as the tumor bulk, where more than $90 \%$ of cells were of human origin. The nucleolin-positive human cells in normal mouse tissue with the maximum distance from the tumor edge/margin were identified, and their distances from the tumor edge/margin were measured $(n=10)$. The statistical significance of these distances was analyzed using Prism 6 software (GraphPad).

\section{Histopathological Staining and Quantification of Invasion, Proliferation, and Microvessel Density}

Paraffin-embedded mouse brains were cut into 5- $\mathrm{mm}$ slices, deparaffinized in xylene, rehydrated in a descending alcohol series, and stained with $\mathrm{H} \& \mathrm{E}$ according to standard protocols. Other slices were deparaffinized in the same manner and probed with mouse anti-nucleolin, rabbit anti-Ki-67, and rabbit anti-CD31 (all Abcam) primary antibodies overnight at $4^{\circ} \mathrm{C}$. The slides were then incubated with polyclonal secondary antibodies and exposed to peroxidase (both Abcam) and DAB (3,3'-diaminobenzidine) substrate (Vector Laboratories), according to the manufacturers' protocols. Counterstaining with hematoxylin was performed according to established standards, 
and the probes were evaluated with an Olympus BX51 microscope. Invasion, proliferation, and microvessel density were quantified as described previously. ${ }^{25}$ In brief, random fields of 10 samples at a $\times 20$ magnification for each condition were chosen and quantified. For quantification of Ki67-positive cells, cells on immunohistochemical images stained with Ki-67-specific antibody were counted using ImageJ software. For total cell count, the images were converted to 8-bit, and the threshold was adjusted to account for all cells in the image. Then, using Binary Process in ImageJ, total cell counts were calculated. For Ki-67-positive cell counts, brightness thresholds were set to minimum $=0$ and maximum $=143$ to facilitate background reduction, and stained $\mathrm{Ki}-67$-positive cells were counted using cell counter plugin of ImageJ software. The ratio of $\mathrm{Ki}-67-$ positive/total cell counts is shown in the figures.

\section{Clinical Outcome Analyses and Analysis of the Gene Signature}

Genes that were common to the 3 cellular and biological functions (i.e., proliferation, invasion, and angiogenesis) were selected to be included in the dexamethasoneregulated core gene signature $(\mathrm{n}=36)$. Expression data were available for 33 genes of the 36 among TCGA patients $(n=520)$; the 3 remaining genes were excluded. Patients were grouped according to whether they had a high or low dexamethasone signature on the basis of individual genes' median expression levels across the patient cohort. A Kaplan-Meier curve analysis of patients with high $(\mathrm{n}=252)$ and low $(\mathrm{n}=263)$ dexamethasone signatures was performed using GraphPad Prism 6 software; 5 of the 520 patients were censored due to lacking data. A log-rank (Mantel-Cox) test was used to calculate the $\mathrm{p}$ value. A heat map for the gene expression levels across patients was generated using GENE-E software from the Broad Institute, which converts values to heat map colors using the mean and maximum values for each row or the standard deviations from the mean for each row.

\section{Results}

\section{A Dexamethasone-Regulated Network Is Activated in Mesenchymal GBM and Can Promote Proliferation, Invasion, and Angiogenesis}

We compared TCGA GBM patient expression profiles of the mesenchymal subgroup $(n=155)$ with those of patients with GBM of the proneural subgroup $(\mathrm{n}=$ 99) (top 50 up- and 50 downregulated genes are shown in Supplemental Table 1). Three different gene lists with increasing stringency of fold-change cutoff, i.e., 5, 6, and 8 , of differentially expressed genes were used as input for analysis with IPA. This analysis revealed that expression levels (upregulated/downregulated) of genes potentially regulated by dexamethasone predict an activated status of dexamethasone among others shown in red across the 3 input gene sets in Fig. 1A, whereas expression levels of genes potentially regulated by actinomycin $\mathrm{D}$ predict an inhibited status of actinomycin D shown in blue across the 3 input gene sets in Fig. 1A. The specific genes (and their relationship to dexamethasone) whose differential expression in mesenchymal versus proneural GBM patients pre- dicted activated status of dexamethasone are shown in Fig. 1B (activation z-score 2.58, $\mathrm{p}=7.59 \mathrm{E}-17$ ) (Fig. 1B and Supplemental Table 2). Furthermore, the comparison of differentially expressed genes in the TCGA mesenchymal subgroup versus proneural subgroup predicts activation of various key cellular functions, such as cell proliferation (activation z-score 1.793, $\mathrm{p}=1.50 \mathrm{E}-38$ ), invasion (activation $\mathrm{z}$-score $3.586, \mathrm{p}=8.99 \mathrm{E}-13$ ), and angiogenesis (activation $\mathrm{z}$-score $4.445, \mathrm{p}=3.03 \mathrm{E}-12$ ). Venn diagrams are an unbiased way of showing the association of differentially regulated genes in mesenchymal versus proneural analysis to cellular functions with activated z-scores, i.e., proliferation, invasion, and angiogenesis (Fig. 1C). The Venn diagram illustrates the numbers of genes and their association with their known cellular functions. We further identified that some of these genes have known overlapping roles across 3 cellular functions (Fig. 1C and Supplemental Table 3).

To evaluate the direct role of dexamethasone in promoting these cellular functions, we treated patient-derived orthotopic GSC3 and GSC6 tumor-bearing mice with dexamethasone, as shown in Fig. 1D. The cells were enriched with FACS from orthotopic tumors obtained in control (Fig. 1E) and dexamethasone-treated (Fig. 1F) mice. We obtained whole genome gene expression profiles for all the tested conditions and then performed GSEA using genes from ex vivo GSC3 dexamethasone versus control expression profiles against the "hallmarks" gene matrix (Fig. $1 \mathrm{G})$. We observed significant enrichment of epithelial-tomesenchymal transition in GSC3 dexamethasone-treated samples, with a normalized enrichment score of $1.52(\mathrm{p}$ $=0.0024 ;$ FDR q-value $=0.056$ ). A network analysis was performed using significantly altered genes (mRNAs and miRNAs) and a fold cutoff of \pm 1.5 ; similar cellular functions-proliferation (z-score 3.521, $\mathrm{p}=6.18 \mathrm{E}-10$ ), invasion (z-score 4.105, $\mathrm{p}=1.69 \mathrm{E}-07$ ), and angiogenesis (z-score $2.202, \mathrm{p}=5.44 \mathrm{E}-05)$ - were activated in dexamethasonetreated samples as in control samples (Fig. $1 \mathrm{H}$ and Supplemental Table 3).

\section{Dexamethasone Promotes Cell Invasion In Vitro and In Vivo}

To establish a link between dexamethasone exposure of GSCs and cell invasion in GBM, we evaluated the invasion potential of GSCs in vitro by comparing the frequencies of invading cells in dexamethasone-treated and control groups. The numbers of invading GSC3 and GSC6 cells were higher in the dexamethasone-treated group (Fig. 2A and Supplementary Fig. 1B). When the numbers of invading cells were quantified, a significantly higher number of invading GSC3 (i.e., IDHI wild type) cells were found in the dexamethasone-treated group than in the control group $(\mathrm{p}=0.02)$ (Fig. 2B); only an increasing trend was found for GSC6 (i.e., IDH1 mutant) $(\mathrm{p}=0.09)$ (Supplementary Figs. 1A and C). Similarly, an analysis of the invading edges of orthotopic tumors from dexamethasone-treated and control mice showed that cells of human origin (marked by the human-specific nucleolin antibody) invaded longer distances into normal mouse brain tissue in dexamethasone-treated mice than in control mice (Fig. $2 \mathrm{C}$ and Supplementary Fig. 1D); these cells are quanti- 


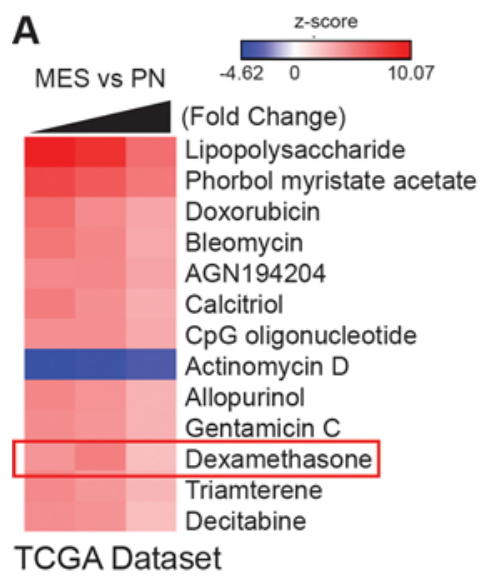

TCGA Dataset
B

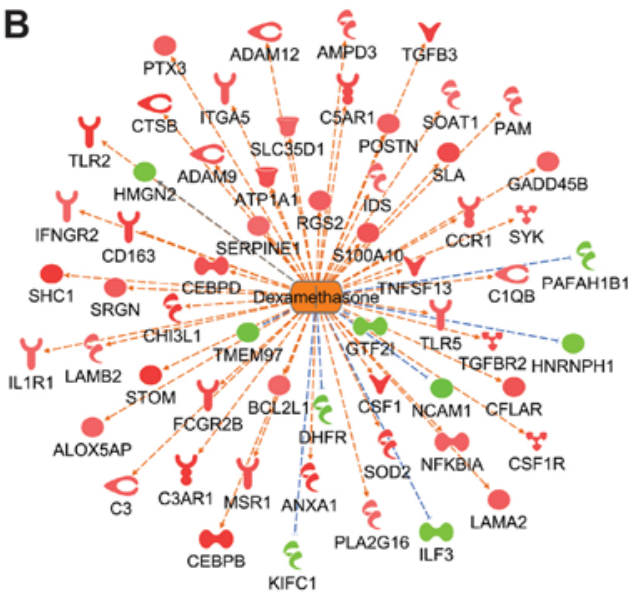

Dexamethasone Network

(z-score: 2.58; p-value: 7.59E-17)

TCGA Mesenchymal vs Proneural

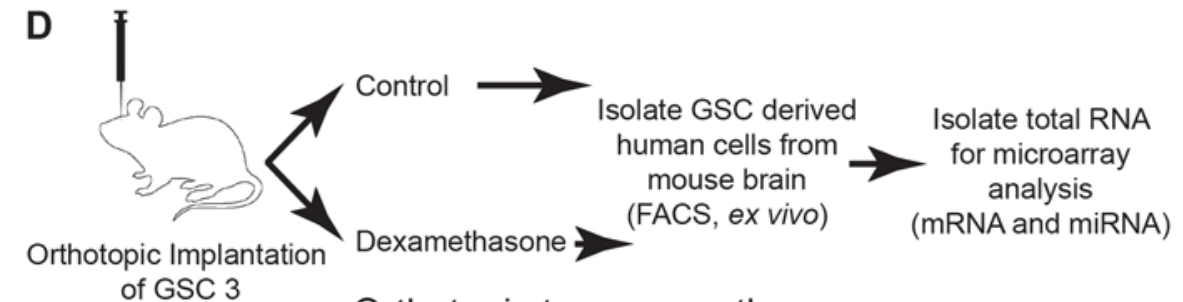
of GSC 3

\section{Orthotopic tumor growth}

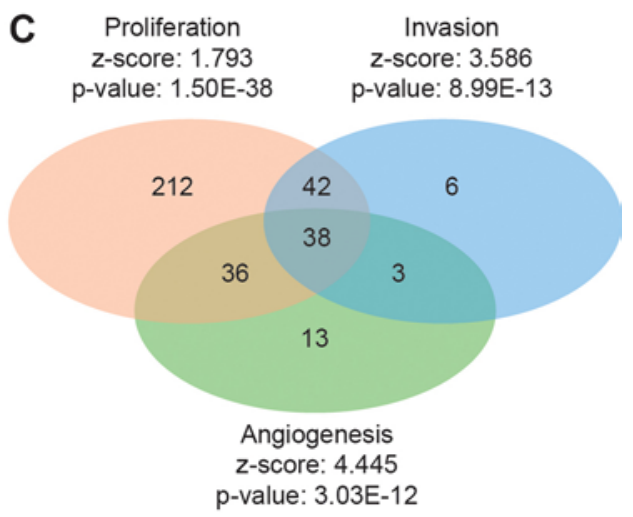

TCGA Mesenchymal vs Proneural

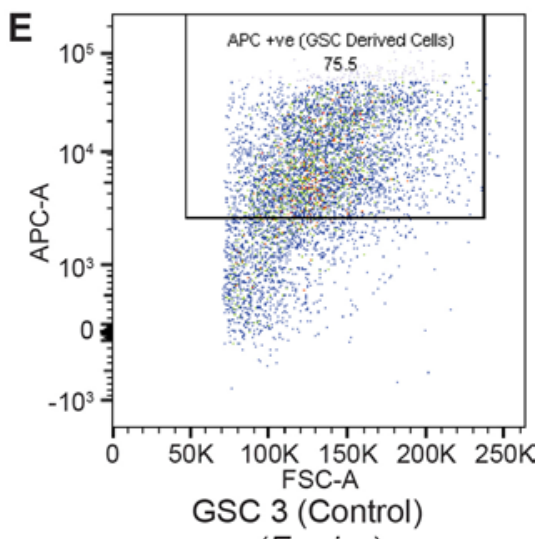

(Ex vivo)
$\mathbf{F}$

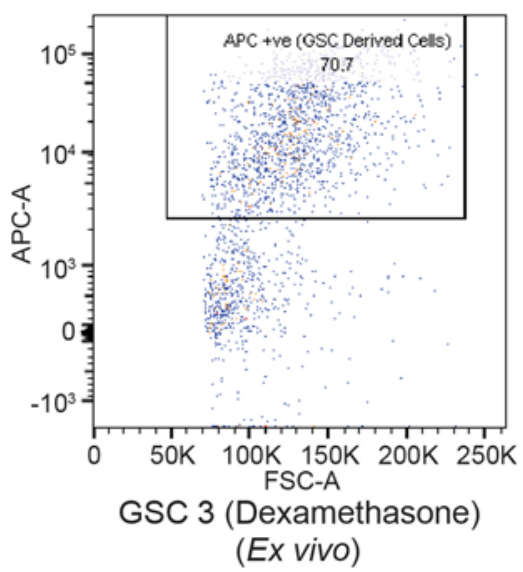

G

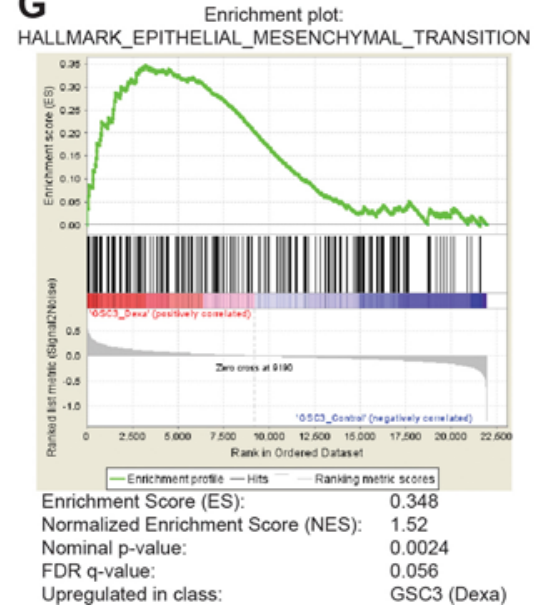

H

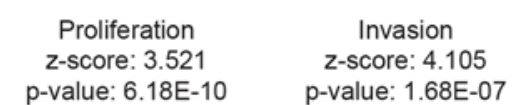
p-value: $1.68 \mathrm{E}-07$

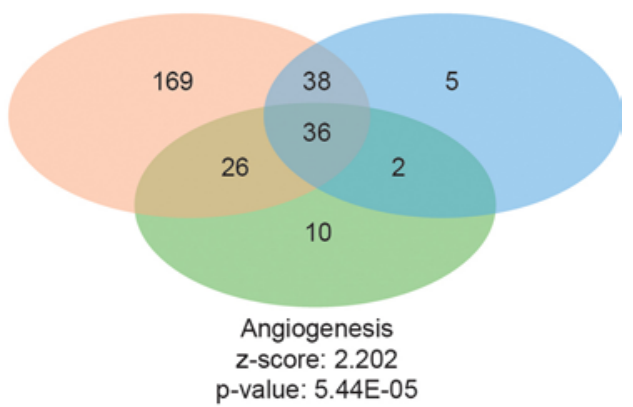

GSC3 Dexamethasone vs Control (Ex vivo)

FIG. 1. Dexamethasone promotes a protumorigenic gene signature in GBM. A: Heat map depicting z-scores of activation (red) and inhibition (blue) for various drugs, illustrating the status of these various drugs and their downstream regulated gene networks in mesenchymal (MES) GBM patients compared with proneural (PN) GBM patients. Fold-change cutoffs from left to right of the heat map are 5, 6, and 8 in MES versus PN comparisons. B: Activated dexamethasone-regulated gene network contributing to the prediction of the activated z-score for the dexamethasone-regulated gene network and its differential expression in MES compared with PN GBM patients. The z-score and the $p$ value of activation are shown below the network. C: Venn diagram of genes from the MES versus PN comparison showing association of differentially regulated genes in MES vs PN analysis to various activated cellular functions, i.e., proliferation, invasion, and angiogenesis. The z-score and the $p$ value of activation are presented under each cellular function. The numbers represent the numbers of genes involved. D: Schematic representation of the experimental plan to investigate the role of dexamethasone in GSC-derived orthotopic tumors, as well as downstream ex vivo analyses. $E$ and F: Ex vivo enrichment of GSC3-derived cells of human origin from control (E) and dexamethasone-treated (F) mouse brains. G: Gene set enrichment plot for epithelial to mesenchymal transition in ex vivo GSC3 dexamethasone-treated samples. Values for the enrichment score, normalized enrichment score, nominal $\mathrm{p}$ value, FDR, and class identification are shown under the plot. H: Venn diagram of altered genes in dexamethasone-treated versus control ex vivo cells showing their cellular function association. The $z$-score and $p$ value of activation are presented under each cellular function. 
A

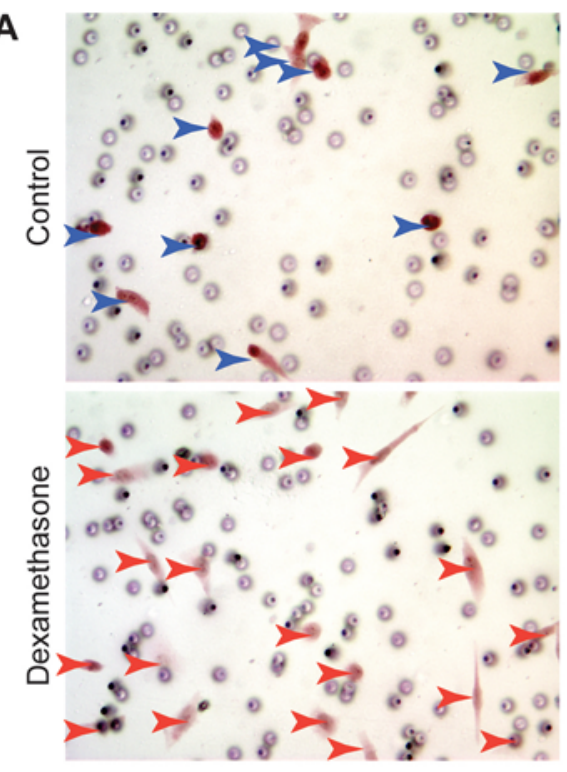

C GSC3 Derived Orthotopic Tumor

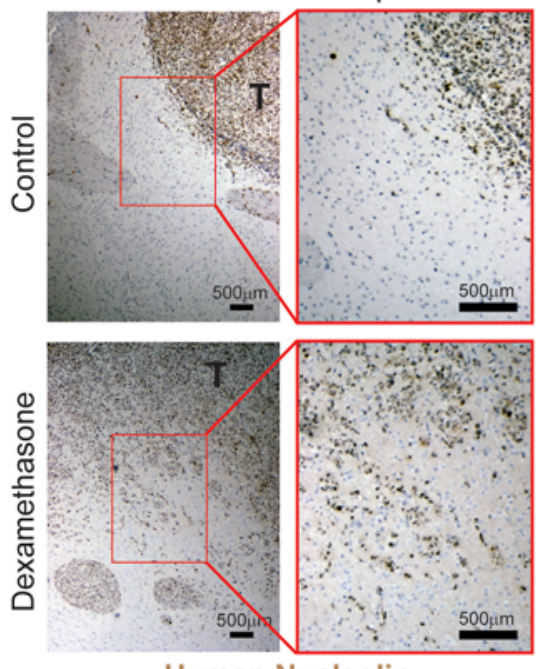

B

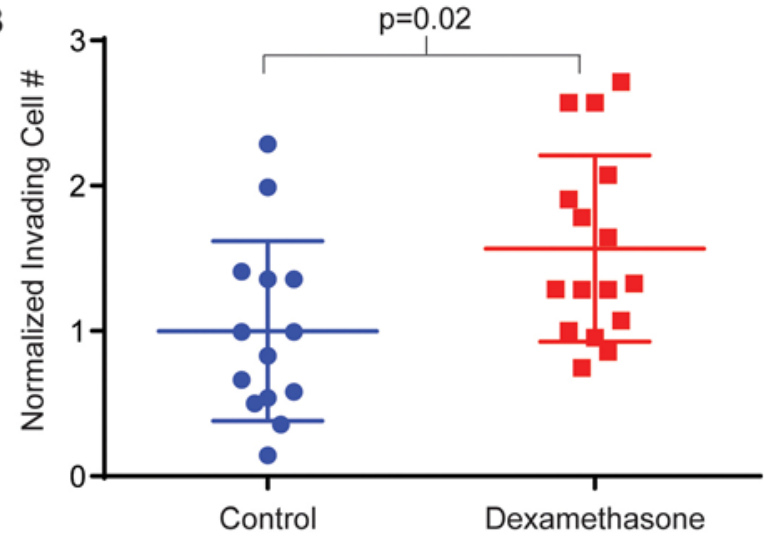

D

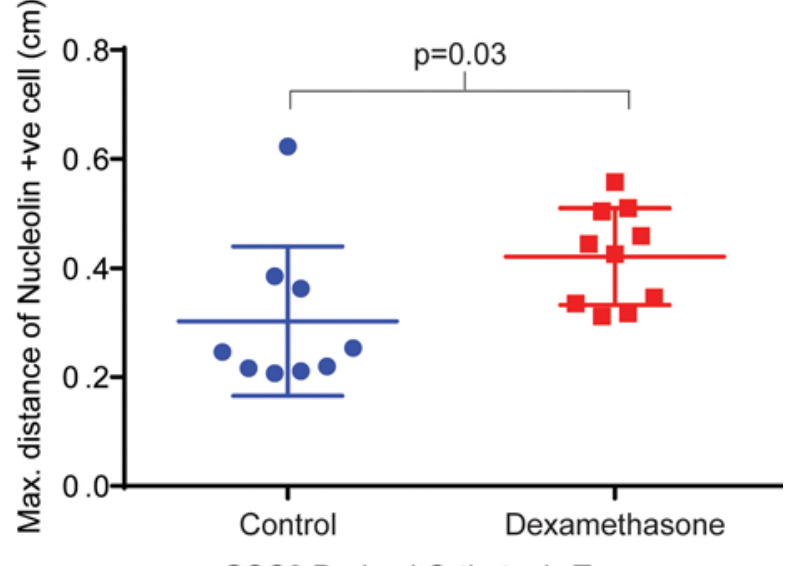

GSC3 Derived Orthotopic Tumor

Human Nucleolin

FIG. 2. Dexamethasone promotes cell invasion in vitro and in vivo. A and B: Dexamethasone promotes cell invasion in GSC3 in vitro. Representative micrographs of control and dexamethasone-treated H \& E-stained Boyden chamber membranes show cells (arrowheads) that have invaded through the Matrigel layer and are present on the other side of membrane (A). Quantification of invading cells in control and dexamethasone-treated membranes is shown in panel B. Error bars represent the standard deviation around the mean value, and the $p$ value is shown above the graph. $\mathbf{C}$ and $\mathbf{D}$ : Dexamethasone treatment promotes the in vivo invasion of GSC3-derived orthotopic tumors. Micrographs of human-specific nucleolin-stained mouse brains with orthotopic tumors, including a representative low magnification (left) and high magnification (right; boxed in red within the left panel) to show the tumor margin (C). The bulk of the tumor is marked with "T" in the left panels. Scale bars are shown in the micrograph. Quantification of invading human nucleolin-positive cells in normal mouse brain away from the bulk of the tumor (D). Error bars represent the standard deviation around the mean value, and the $p$ value is shown above the graph. The dots and squares represent normalized numbers of invading cells.

fied in Fig. 2D and Supplementary Fig. 1E for GSC3s ( $\mathrm{p}=$ $0.03)$ and GSC6s $(\mathrm{p}=0.24)$, respectively.

\section{Dexamethasone Promotes Cell Proliferation and Angiogenesis In Vitro and In Vivo}

Our in vivo results show that dexamethasone treatment resulted in increased cell proliferation and angiogenesis in both GSC3- (IDH1 wild type) and GSC6- (IDH1 mutant) derived tumors. To assess cell proliferation, we stained or- thotopic tumor-bearing sections of mouse brains with Ki67 (proliferation marker). As shown in the representative micrographs, a significantly higher ratio of Ki-67-positive/total cells was found in dexamethasone-treated mice in both GSC3- (p < 0.0001) (Fig. 3A and B) and GSC6- (p $=0.0001$ ) derived orthotopic tumors (Fig. $3 \mathrm{C}$ and D).

We evaluated the status of the tumor vasculature in dexamethasone-treated tumors and compared it to that of control tumors. As shown in the representative micro- 


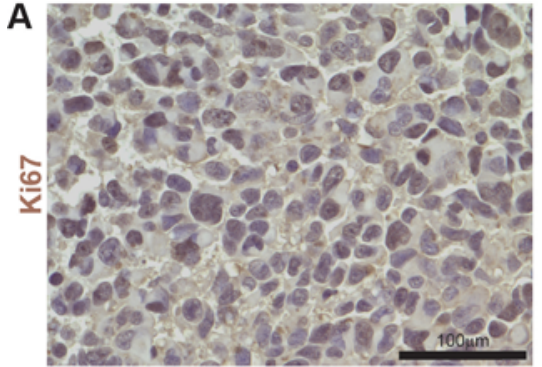

Control (GSC3)

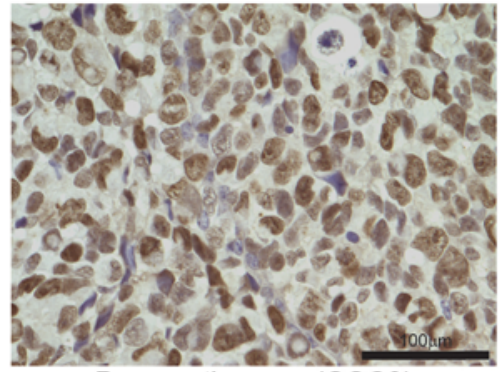

Dexamethasone (GSC3)

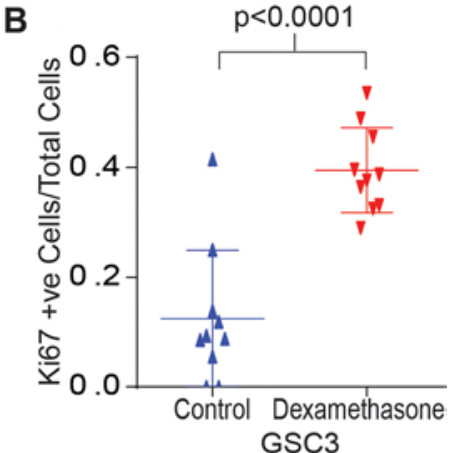

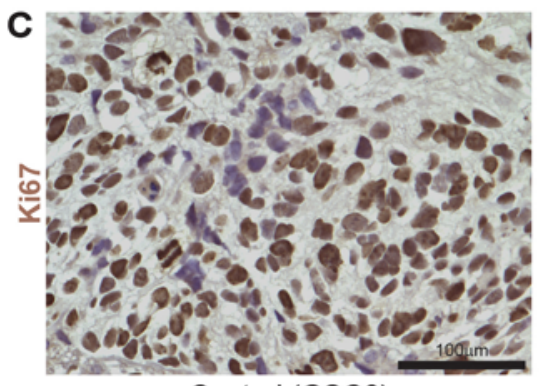

Control (GSC6)

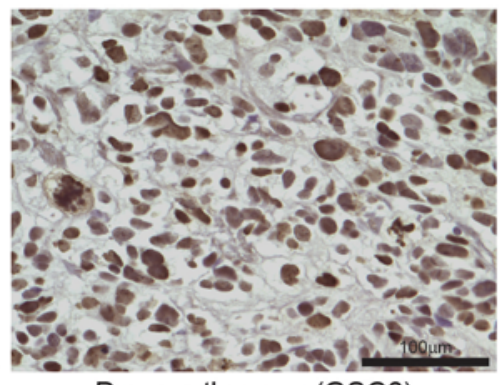

Dexamethasone (GSC6)

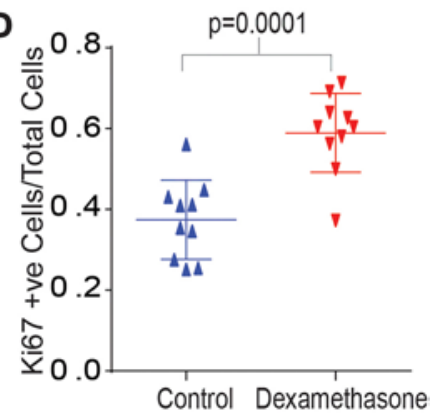

GSC6

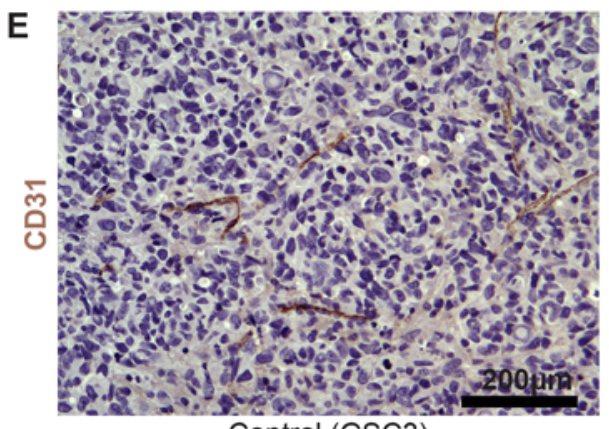

Control (GSC3)

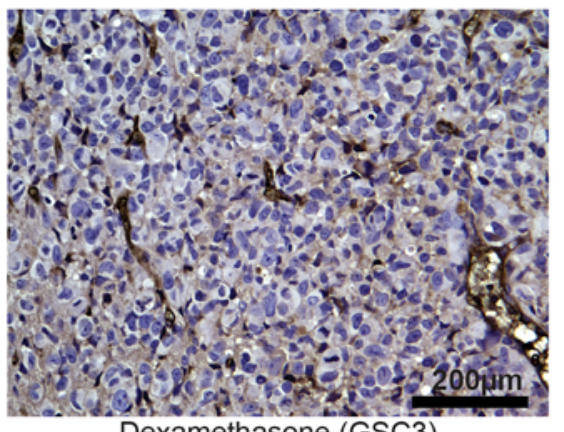

Dexamethasone (GSC3)

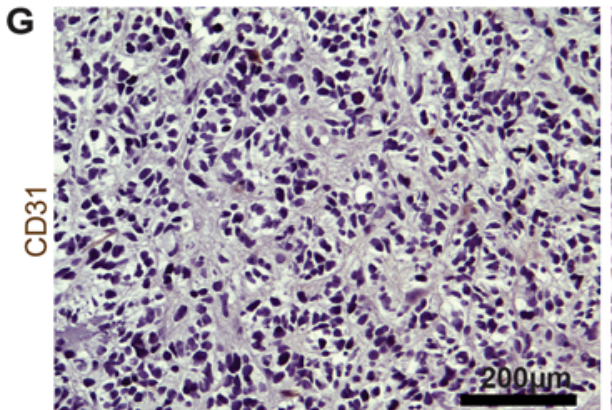

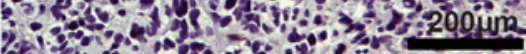

Control (GSC6)

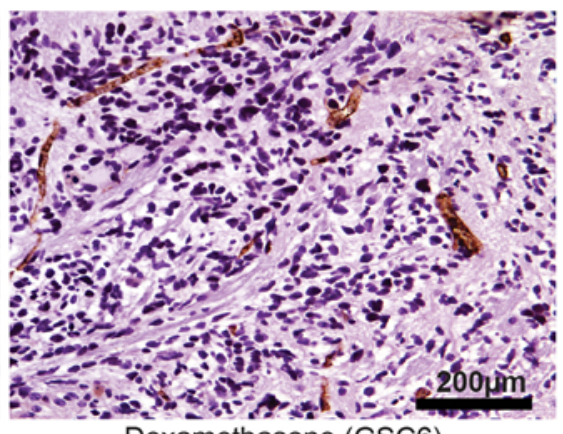

Dexamethasone (GSC6)
F Control

- Dexamethasone

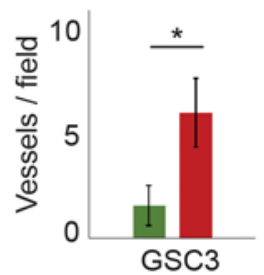

FIG. 3. Dexamethasone promotes cell proliferation and angiogenesis in vivo. A and C: Representative micrographs of proliferation marker Ki-67-stained orthotopic control and dexamethasone-treated tumors derived from GSC3 (A) and GSC6 (C). Bar = $100 \mu \mathrm{m}$ in A and C. B and D: Quantification of ratio of Ki-67-positive/total cells in control and dexamethasone-treated GSC3 (B) and GSC6 (D) tumors. Error bars represent the standard deviation around the mean value, and the $p$ value is shown above the graph. E and G: Representative micrographs of endothelial marker CD31-stained orthotopic control and dexamethasone-treated tumors derived from GSC3 (E) and GSC6 (G). Bar $=200 \mu \mathrm{m}$ in E and G. F and H: Quantification of CD31-positive cells in control and dexamethasone-treated GSC3 (F) and GSC6 $(H)$ tumors. Error bars represent the standard deviation around the mean value. The triangles represent normalized numbers of invading cells. ${ }^{*} \mathrm{p}=1.16 \mathrm{E}-07$ in $\mathrm{F}$ and $9.49 \mathrm{E}-09$ in $\mathrm{H}$. 


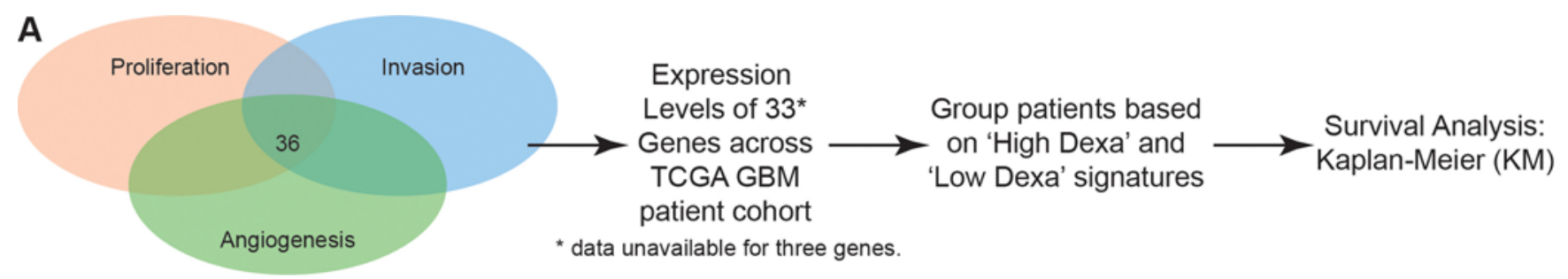

GSC3 Dexamethasone vs Control

(Ex vivo)
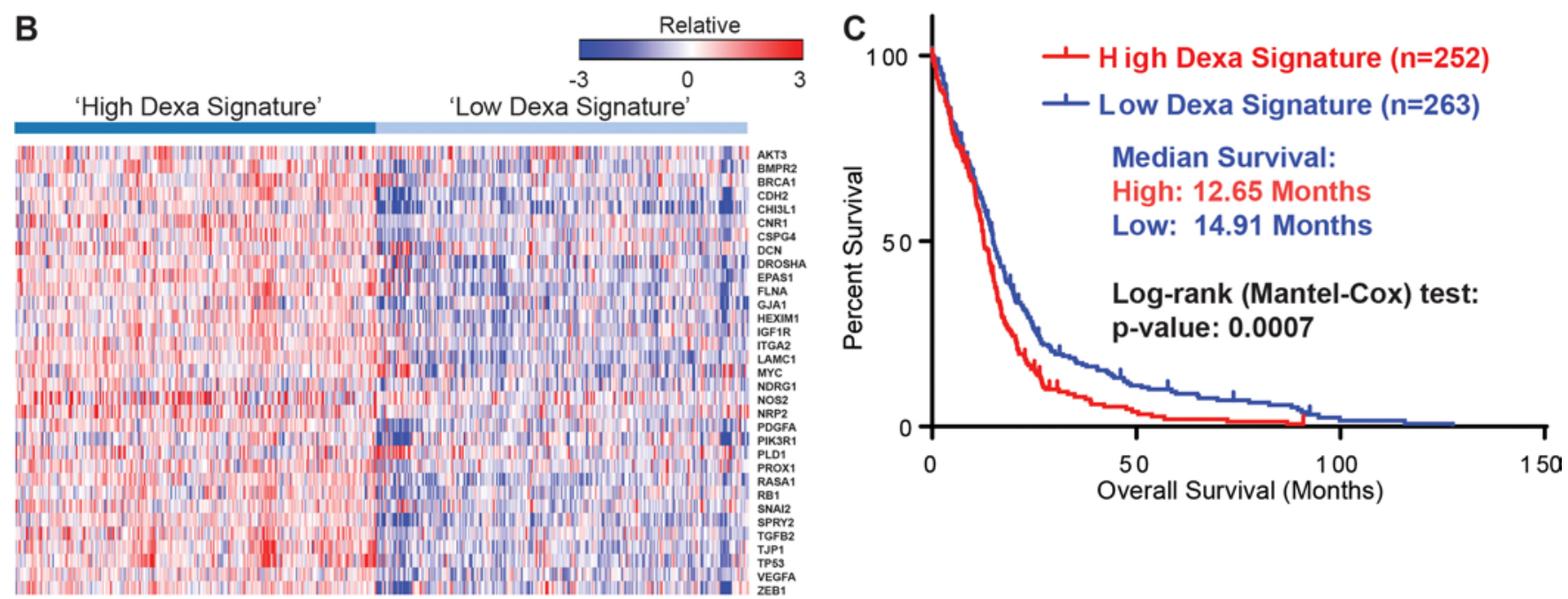

TCGA $(n=520)$

FIG. 4. Dexamethasone (dexa)-promoted gene signature has prognostic value for GBM patients. A: Schematic representation of the derivation of the dexamethasone-regulated gene signature from an ex vivo expression profile analysis, subgrouping of TCGA patients, and a survival analysis. B: Heat map showing the expression levels of 33 genes among TCGA patients $(n=520)$ with a high or low dexamethasone signature. The color index bar is shown above the heat map. C. Kaplan-Meier curve analysis of patients with high and low dexamethasone signatures. The median survival durations in months are shown within the plot.

graphs, the CD31 (endothelial cell marker) signal is significantly higher in dexamethasone-treated orthotopic tumors, indicating an increase in angiogenesis on dexamethasone treatment in both GSC3- (Fig. 3E) and GSC6- (Fig. 3G) derived tumors. We quantified the numbers of CD31-positive cells in 3 independent microscopic fields and found a statistically significant increase in both GSC3- (Fig. 3F; $\mathrm{p}=1.16 \mathrm{E}-07$ ) and GSC6- (Fig. 3H; $\mathrm{p}=9.49 \mathrm{E}-09$ ) derived tumors.

\section{Gene Expression Analysis of Dexamethasone Versus Sham-Treated Orthotopic Tumors Reveals a Prognostic Gene Signature in a Large Cohort of GBM Patients}

Furthermore, in an effort to determine the clinical relevance of our ex vivo-derived dexamethasone-regulated genes, we selected the 36 genes with established and overlapping roles in cell proliferation, cell invasion, and angiogenesis from the dexamethasone versus sham-treated orthotopic tumors (Fig. 4A and Supplemental Tables 4 and 5). Of these 36 genes, the TCGA data set contains expression values for 33 genes. Ingenuity pathway analysis of these 33 genes revealed "glioblastoma multiforme signaling" as the top altered canonical pathways $(p=5.43 \mathrm{E}-09)$ (Supplemental Table 6). On the gene level, the 3 prooncogenic genes $A K T 3, B R C A 1$, and $M Y C$ appeared to be part of the 33 ex vivo-derived dexamethasone-regulated genes. We then performed a Kaplan-Meier curve analysis of the entire TCGA cohort of GBM patients $(n=520)$. Patients were dichotomized into high and low dexamethasone signature groups (Fig. 4B). The survival differences between these 2 groups were evaluated by Kaplan-Meier curve analysis. As shown in Fig. 4C, patients with a high dexamethasone signature $(n=252)$ had significantly shorter overall survival durations ( $\mathrm{p}=0.0007$; median 12.65 months) than those with a low signature $(n=263$; median 14.91 months).

\section{Discussion}

In this article, we present evidence suggesting that dexamethasone induces invasion, proliferation, and angiogenesis in patient-derived GSC lines both in vitro and in vivo.

Diffuse invasion, proliferation, and abundant angiogenesis are hallmarks of $\mathrm{GBM}^{7}$ and are major drivers of its gross morphological characteristics, clinical aggressiveness, and dismal patient outcome. The genes of the more aggressive mesenchymal and inflammatory subtype of GBM may directly or indirectly contribute to the activated status of invasion, proliferation, and angiogenesis. ${ }^{11}$ 
The mesenchymal GBM expression profiles include altered gene networks that predicted an activated status of dexamethasone, a drug that is routinely used to manage edema in GBM patients. Our results further elucidated the presence of a link between dexamethasone exposure and alterations in gene expression profiles, which resulted in activation of invasion, proliferation, and angiogenesis in GBM cell lines and orthotopic xenograft models. In addition, with our gene expression analysis of dexamethasone versus sham-treated orthotopic tumors, we identified a 33gene signature that was prognostic of overall survival in GBM patients.

Interestingly, our data also demonstrated in vivo and in vitro that dexamethasone promoted invasion, proliferation, and in vivo it promoted angiogenesis in an $\mathrm{IDH} 1$ mutant cell line; the latter mutation is frequently associated with secondary, less vascularized GBMs. Our findings demonstrated that dexamethasone indeed promotes cell invasion, cell proliferation, and angiogenesis across distinct GBM subtypes, although these effects are more pronounced in more aggressive cells such as GSC3, which is derived from a patient who harbored a mesenchymal and very inflammatory GBM subtype. Vascular endothelial growth factor (VEGF), which is an orchestrator of angiogenesis and perilesional vasogenic edema, was proposed as a therapeutic target in GBM. ${ }^{23}$ It follows that bevacizumab-treated GBM patients were shown to require less dexamethasone since anti-VEGF therapy appeared to reduced vasogenic edema as well., ${ }^{414}$ However, our results suggest that dexamethasone may have genome-wide genetic implications in GBM, and, in addition to the beneficial antiinflammatory effects, it also potentially promotes angiogenesis and other hallmarks of cancer in a more delayed fashion.

Consistent with previously published in vitro findings, ${ }^{11}$ our gene expression analysis of dexamethasone- versus sham-treated orthotopic tumors revealed that genes altered by dexamethasone exposure not only appear to have overlapping cellular functions associated with proliferation, invasion, and angiogenesis, but also were prognostic of significantly poorer patient survival, illustrating the need for targeted and balanced (with respect to countering negative side effects) antiedema therapy in GBM. One possible therapeutic approach is low-dose camptothecin administration, which is well tolerated. We previously validated this drug's ability to potentially counter dexamethasone-induced oncogenic effects in vitro. ${ }^{11}$

Despite the faithful replication of the human disease, any type of model system has inherent pitfalls. To determine the role of dexamethasone in vivo and to cover the 2 extreme GBM subtypes, we used both IDH1 wild-type (GSC3) and mutant (GSC6) GSC lines. Larger numbers of cell lines will be needed in further confirmatory studies, and ultimately prospective clinical trials will provide a definite answer; however, this is beyond the scope of the current study. Working with xenografts requires nude mice, which lack a thymus; they are thus unable to produce a cell-mediated immune response, which is a limitation of our data since dexamethasone influences immunological pathways,${ }^{16}$ and tumor's ability to evade immune destruction is an emerging hallmark of cancer. ${ }^{6}$ This might be a limitation considering the study's relevance and application to clinical practice, yet our study design also mini- mizes potential confounding effects of immune/inflammatory responses created in response to dexamethasone. Future research has to prove our findings in syngenic or sister xenograft models. In addition, it is possible that the more aggressive tumors studied in the TCGA also received more steroid treatment; nevertheless, the poorer survival of patients carrying tumors with a dexamethasone-induced gene signature lends further support to our in vitro and in vivo findings. In addition, the correlation of patient survival duration and dexamethasone dose has been established in a recent clinical study ${ }^{27}$ thus further, at least in part, supporting our findings. This effort to determine the clinical relevance of our ex vivo xenograft microarray-derived dexamethasone-regulated genes with Kaplan-Meier curve analysis revealed that known regulators of oncogenicity, such as AKT3, BRCA1, and MYC, are affected by dexamethasone exposure. AKT3 was previously shown to play a significant role in GBM viability, ${ }^{13}$ and respective knockdown was associated with decreased invasiveness of GBM cells. ${ }^{18}$ Interestingly, the tumor suppressor BRCA1 has recently been shown to promote tumorigenicity in GBM, ${ }^{20}$ while the proto-oncogene $M Y C$ is well known to play a role in GBM.

\section{Conclusions}

We present evidence that furthers our understanding of the complex effects of dexamethasone on biological characteristics of GBM. The results suggest that the drug increases invasion, proliferation, and angiogenesis in human GSC-derived orthotopic tumors, potentially worsening GBM patients' prognoses. We believe that further careful investigation is needed to determine how to minimize these deleterious dexamethasone-mediated side effects in glioblastoma.

\section{Acknowledgments}

Supported by NIH grant no. 1P50 CA12CA127001 to Drs. Sulman and Lang.

\section{References}

1. Campana D, Manabe A, Evans WE: Stroma-supported immunocytometric assay (SIA): a novel method for testing the sensitivity of acute lymphoblastic leukemia cells to cytotoxic drugs. Leukemia 7:482-488, 1993

2. Carro MS, Lim WK, Alvarez MJ, Bollo RJ, Zhao X, Snyder EY, et al: The transcriptional network for mesenchymal transformation of brain tumours. Nature 463:318-325, 2010

3. Fu J, Khaybullin R, Liang X, Morin M, Xia A, Yeh A, et al: Discovery of gene regulation pattern in lung cancer by gene expression profiling using human tissues. Genom Data 3:112-115, 2015

4. Gilbert MR, Dignam JJ, Armstrong TS, Wefel JS, Blumenthal DT, Vogelbaum MA, et al: A randomized trial of bevacizumab for newly diagnosed glioblastoma. N Engl J Med 370:699-708, 2014

5. Hanahan D, Weinberg RA: The hallmarks of cancer. Cell 100:57-70, 2000

6. Hanahan D, Weinberg RA: Hallmarks of cancer: the next generation. Cell 144:646-674, 2011

7. Hardee ME, Zagzag D: Mechanisms of glioma-associated neovascularization. Am J Pathol 181:1126-1141, 2012

8. Hegi ME, Diserens AC, Gorlia T, Hamou MF, de Tribolet N, 
Weller M, et al: MGMT gene silencing and benefit from temozolomide in glioblastoma. N Engl J Med 352:997-1003, 2005

9. Hossain A, Gumin J, Gao F, Figueroa J, Shinojima N, Takezaki T, et al: Mesenchymal stem cells isolated from human gliomas increase proliferation and maintain stemness of glioma stem cells through the IL-6/gp130/STAT3 pathway. Stem Cells 33:2400-2415, 2015

10. Jain RK, di Tomaso E, Duda DG, Loeffler JS, Sorensen AG, Batchelor TT: Angiogenesis in brain tumours. Nat Rev Neurosci 8:610-622, 2007

11. Luedi MM, Singh SK, Mosley JC, Hatami M, Gumin J, Sulman EP, et al: A dexamethasone-regulated gene signature is prognostic for poor survival in glioblastoma patients. J Neurosurg Anesthesiol 29:46-58, 2017

12. Moroz MA, Huang R, Kochetkov T, Shi W, Thaler H, de Stanchina E, et al: Comparison of corticotropin-releasing factor, dexamethasone, and temozolomide: treatment efficacy and toxicity in U87 and C6 intracranial gliomas. Clin Cancer Res 17:3282-3292, 2011

13. Mure H, Matsuzaki K, Kitazato KT, Mizobuchi Y, Kuwayama K, Kageji T, et al: Akt2 and Akt3 play a pivotal role in malignant gliomas. Neuro Oncol 12:221-232, 2010

14. Nghiemphu PL, Liu W, Lee Y, Than T, Graham C, Lai A, et al: Bevacizumab and chemotherapy for recurrent glioblastoma: a single-institution experience. Neurology 72:1217-1222, 2009

15. Nobusawa S, Watanabe T, Kleihues P, Ohgaki H: IDH1 mutations as molecular signature and predictive factor of secondary glioblastomas. Clin Cancer Res 15:6002-6007, 2009

16. Oppong E, Cato AC: Effects of glucocorticoids in the immune system. Adv Exp Med Biol 872:217-233, 2015

17. Parsons DW, Jones S, Zhang X, Lin JC, Leary RJ, Angenendt $\mathrm{P}$, et al: An integrated genomic analysis of human glioblastoma multiforme. Science 321:1807-1812, 2008

18. Paul-Samojedny M, Pudełko A, Suchanek-Raif R, Kowalczyk M, Fila-Daniłow A, Borkowska P, et al: Knockdown of the AKT3 (PKB $\gamma)$, PI3KCA, and VEGFR2 genes by RNA interference suppresses glioblastoma multiforme T98G cells invasiveness in vitro. Tumour Biol 36:3263-3277, 2015

19. Phillips HS, Kharbanda S, Chen R, Forrest WF, Soriano RH, Wu TD, et al: Molecular subclasses of high-grade glioma predict prognosis, delineate a pattern of disease progression, and resemble stages in neurogenesis. Cancer Cell 9:157-173, 2006

20. Rasmussen RD, Gajjar MK, Tuckova L, Jensen KE, MayaMendoza A, Holst CB, et al: BRCA1-regulated RRM2 expression protects glioblastoma cells from endogenous replication stress and promotes tumorigenicity. Nat Commun 7:13398, 2016

21. Reya T, Morrison SJ, Clarke MF, Weissman IL: Stem cells, cancer, and cancer stem cells. Nature 414:105-111, 2001

22. Singh SK, Hawkins C, Clarke ID, Squire JA, Bayani J, Hide $\mathrm{T}$, et al: Identification of human brain tumour initiating cells. Nature 432:396-401, 2004

23. Stratmann A, Machein MR, Plate KH: Anti-angiogenic gene therapy of malignant glioma. Acta Neurochir Suppl 68:105-110, 1997

24. Verhaak RG, Hoadley KA, Purdom E, Wang V, Qi Y, Wilkerson MD, et al: Integrated genomic analysis identifies clinically relevant subtypes of glioblastoma characterized by abnormalities in PDGFRA, IDH1, EGFR, and NF1. Cancer Cell 17:98-110, 2010
25. Weidner N, Semple JP, Welch WR, Folkman J: Tumor angiogenesis and metastasis-correlation in invasive breast carcinoma. N Engl J Med 324:1-8, 1991

26. Wen PY, Kesari S: Malignant gliomas in adults. N Engl J Med 359:492-507, 2008

27. Wong ET, Lok E, Gautam S, Swanson KD: Dexamethasone exerts profound immunologic interference on treatment efficacy for recurrent glioblastoma. Br J Cancer 113:232-241, 2015

28. Yan H, Parsons DW, Jin G, McLendon R, Rasheed BA, Yuan W, et al: IDH1 and IDH2 mutations in gliomas. N Engl J Med 360:765-773, 2009

\section{Disclosures}

Dr. Sulman: non-study-related clinical support from Novocure and AbbVie. Funding was provided by the following. Dr. Luedi: The Mach-Gaensslen Foundation Neurology Grant 2017 from the Mach-Gaensslen Foundation, Unteraegeri, Switzerland; Alfred \& Anneliese Sutter-Stoettner Foundation, Muenchwilen, Switzerland; and Foundation for Research in Anesthesiology and Intensive Care Medicine, Bern, Switzerland. Dr. Colen: John S. Dunn Sr. Distinguished Chair in Diagnostic Imaging and The University of Texas MD Anderson Cancer Center Start-up Fund. Dr. Zinn: Neurosurgery Research \& Education Foundation.

\section{Author Contributions}

Conception and design: Zinn, Luedi, Singh, Hatami, Gumin, Andereggen, Sulman, Lang, Stueber, Fuller, Colen. Acquisition of data: Zinn, Luedi, Singh, Mosley, Hassan, Hatami, Gumin, Sulman, Lang, Fuller, Colen. Analysis and interpretation of data: Zinn, Luedi, Singh, Mosley, Hassan, Andereggen, Stueber, Colen. Drafting the article: Zinn, Luedi, Singh, Stueber, Colen. Critically revising the article: Zinn, Luedi, Singh, Andereggen, Colen. Reviewed submitted version of manuscript: all authors. Approved the final version of the manuscript on behalf of all authors: Zinn. Statistical analysis: Zinn, Singh, Hatami. Administrative/techni$\mathrm{cal} /$ material support: Zinn, Luedi, Singh, Mosley, Hatami, Sulman. Study supervision: Zinn, Singh, Colen.

\section{Supplemental Information}

\section{Online-Only Content}

Supplemental material is available with the online version of the article.

Supplemental Figure and Tables. https://thejns.org/doi/suppl/ 10.3171/2017.7.JNS17668.

\section{Previous Presentations}

Portions of this paper (Fig. 3G and H) were presented as abstracts at the Bern University Hospital Inselspital Department of Clinical Research Day of Clinical Research, Bern, Switzerland, November 2, 2016, and The European Society of Anaesthesiology Congress, London, United Kingdom, May 28-30, 2016.

\section{Correspondence}

Pascal O. Zinn: Baylor College of Medicine, Houston, TX. zinn@ bcm.edu. 